\title{
DEPOSIÇÃO DE RESÍDUOS VEGETAIS, MATÉRIA ORGÂNICA LEVE, ESTOQUES DE CARBONO E NITROGÊNIO E FÓSFORO REMANESCENTE SOB DIFERENTES SISTEMAS DE MANEJO NO CERRADO GOIANO(1)
}

\author{
Roni Fernandes Guareschi(2), Marcos Gervasio Pereira ${ }^{(3)}$ \& Adriano Perin ${ }^{(4)}$
}

\begin{abstract}
RESUMO
O sistema plantio direto (SPD), em função de seu tempo de estabelecimento, pode promover aumento na quantidade de resíduos vegetais adicionados à superfície do solo e, consequentemente, modificações nos seus atributos químicos e físicos. O trabalho teve por objetivo quantificar a deposição de resíduos vegetais na superfície do solo (RVS) e as modificações nos teores de matéria orgânica leve (MOL), nos estoques de carbono e nitrogênio, nos teores de fósforo remanescente (Prem) e nos atributos físicos do solo - densidade de partículas (Dp), densidade do solo (Ds) e volume total de poros (VTP) -, bem como avaliar a origem do carbono por meio de técnicas isotópicas $\left({ }^{13} \mathrm{C}\right)$. Para isso, em Montividiu (GO) foram selecionadas áreas sob SPD com diferentes tempos de implantação: SPD com três anos de implantação (SPD3), SPD com 15 anos de implantação (SPD15) e SPD com 20 anos de implantação (SPD20), as quais foram comparadas a uma área de Cerrado nativo stricto sensu (CE) e a uma área de pastagem plantada de Brachiaria decumbens (PA). Em cada uma das áreas, foram coletadas amostras nas profundidades de 0-5, 5-10 e 10-20 cm. O solo das áreas de estudo foi classificado como Latossolo Vermelho distroférrico. $O$ delineamento utilizado foi inteiramente casualizado. Foi observado aumento nos teores de RVS, MOL, VTP, Prem, C e N em função do tempo de implantação do SPD. A área de SPD com 20 anos apresentou maiores valores de $\mathrm{C}$ e $\mathrm{N}$ e valores semelhantes de Ds e MOL, em relação ao CE. As análises de ${ }^{13} \mathrm{C}$ demonstraram que as leguminosas estão contribuindo de forma significativa para a composição da matéria orgância nas áreas sob SPD. Nas áreas sob SPD, verificou-se aumento
\end{abstract}

\footnotetext{
(1) Parte da Tese de Doutorado apresentada, pelo primeiro autor, ao Curso de Pós-Graduação em Agronomia - Ciência do Solo (CPGACS), Universidade Federal Rural do Rio de Janeiro - UFRRJ). Recebido para publicação em 19 de julho de 2011 e aprovado em 6 de março de 2012.

(2) Tecnólogo em Produção de Grãos, Doutorando em Ciência do Solo, Universidade Federal Rural do Rio de Janeiro - UFRRJ. km 7 BR 465, Departamento de Solos, Seropédica (RJ). Bolsista do CNPq. CEP 23890-000 Rio de Janeiro (RJ). E-mail: guareschiecotarelli@ hotmail.com

(3) Professor Associado III do Departamento de Solos, UFRRJ. Bolsista do CNPq e Cientista do Nosso Estado da FAPERJ. E-mail: gervasio@ufrrj.br

(4) Professor do Instituto Federal Goiano - Campus Rio Verde, IF Goiano. Professor do Programa de Pós-Graduação em Ciência Agrárias, IF Goiano. Rod. Sul Goiana Km 01, Zona Rural. CEP 75901-970 Rio Verde (GO). E-mail: perinrj@yahoo.com.br
} 


\begin{abstract}
dos valores de estoque de $\mathrm{C}$ e de $\mathrm{N}$ em função do tempo de implantação em todas as profundidades analisadas; as áreas SPD15 e SPD20 apresentaram nas camadas superficiais valores semelhantes e, ou, superiores aos da área de CE. A área de PA apresentou os piores valores dos atributos analisados, demonstrando estar em estádio mais avançado de degradação.
\end{abstract}

Termos de indexação: plantio direto, pastagem, matéria orgânica do solo.

\author{
SUMMARY: CROP RESIDUES, LIGHT ORGANIC MATTER, CARBON AND \\ NITROGEN STORAGE AND REMAINING PHOSPHORUS \\ UNDER DIFFERENT MANAGEMENT SYSTEMS IN THE \\ CERRADO REGION OF GOIÁS
}

\begin{abstract}
According to the time of management implementation, the amount of crop residues added to the soil surface can increase in no-tillage systems (NTS) and promote changes in the chemical and physical soil properties. The objective of this study was to quantify the deposition of plant residues on the soil surface (RSS) and variations in the light organic matter (LOM), in C and N stocks, remaining phosphorus (Prem), the soil physical characteristics (particle density (PD), bulk density (BD) and total pore volume (TPV)), and to evaluate the origin of $C$ by isotopic techniques $\left({ }^{13} \mathrm{C}\right)$. For this purpose, areas under notillage (NTS) after different periods of implementation were selected in Montividiu (GO) as follow: NTS after 3 years (NTS3), NTS after 15 years (NTS15) and NTS after 20 years of implementation (NTS20). These areas were compared with an area of native cerrado "stricto sensu" (CE) and with a planted pasture of Brachiaria decumbens (PA). In each of these areas, soil samples were collected (layers 0-5, 5-10 and 10-20 cm). The soil of the study areas was classified as Rhodic Hapludox. The experiment was evaluated in a completely randomized design. Increased levels of RSS, LOM, PTV, Prem, C, and N were observed as a function of time of NTS implementation. The NTS20 area showed higher C and N values than the $C E$ area and similar values of $B D$ and $L O M$. The ${ }^{13} C$ analysis showed that legumes contribute significantly to the organic matter content in the NTS areas, where increases in the carbon and nitrogen stocks as function of the time of implementation were also observed in all layers analyzed. In the surface layer of the areas NTS15 and NTS20 these values were similar and, or, higher than under CE. The analyzed properties had poorest values in the PA area, indicating an advanced stage of degradation.
\end{abstract}

Index terms: No-tillage, pasture, soil organic matter.

\section{INTRODUÇÃO}

O Cerrado consiste em uma área sob plena expansão da fronteira agrícola no Brasil, cobrindo aproximadamente 200 Mha (Bustamante et al., 2006), cerca de $23 \%$ do território nacional. Estimativas indicam uma taxa de conversão de vegetação nativa do Cerrado de $1,1 \%$ ao ano, o que equivale a 2,2 Mha ano-1 (Carvalho et al., 2010).

Atualmente, cerca de 80 milhões de hectares do bioma Cerrado vêm sendo cultivados com diferentes usos da terra, o que corresponde a 39,5\% da área total do bioma. As duas classes mais representativas de uso da terra são as pastagens cultivadas e as culturas agrícolas, que ocupam 26,5 e $10,5 \%$ do Cerrado, respectivamente. Cerca de $61 \%$ da área do bioma encontra-se preservada, incluindo áreas com vegetação em regeneração e pastagens nativas (Sano et al., 2008).

Estudos indicam que a derrubada e queima da vegetação nativa, seguida do cultivo do solo no processo de conversão do Cerrado em sistemas de cultivo, resultam em redução dos estoques de carbono (C) do solo e aumento da emissão de gases de efeito estufa (GEE) para a atmosfera (Franchini et al., 2007; Smith et al., 2008). Essa redução no conteúdo de matéria orgânica do solo (MOS) é extremamente prejudicial aos solos de Cerrado, 
uma vez que, segundo Oorts et al. (2003), esse compartimento é responsável por 75 a $85 \%$ da capacidade de troca catiônica (CTC) desses solos, ou seja, a MOS juntamente com a caulinita e os óxidos de ferro e alumínio constituem as superfícies de cargas negativas dos solos de Cerrado (Silva \& Resck, 1997).

Diante do cenário exposto, o sistema plantio direto (SPD) surge como uma alternativa para minimizar os impactos causados ao solo, pelas diferentes formas de uso, pois, segundo Carvalho et al. (2010), a manutenção de resíduos vegetais na superfície, a rotação de culturas e o mínimo revolvimento do solo (revolvimento somente na linha/cova de semeadura), princípios básicos da adoção do SPD, além de reduzirem a emissão de $\mathrm{CO}_{2}$ para a atmosfera, atuam no aumento do estoque de $\mathrm{C}$ e $\mathrm{N}$ no solo. Além disso, o SPD traz ainda outros benefícios, como: aumento da diversidade microbiana, melhoria da fertilidade e dos atributos físicos do solo (Six et al., 2002; Foley et al., 2005) e, ainda, uma expressiva redução na erosão hídrica pelo aumento de resíduos vegetais na superfície do solo (Mello et al., 2006).

No SPD, os solos apresentam, em geral, na camada superficial, após três a quatro anos, maiores valores de densidade e microporosidade e menores valores de macroporosidade e porosidade total, quando comparados com outros sistemas de manejo (Stone \& Silveira, 2001). Esse padrão decorre, principalmente, do arranjamento natural do solo, quando não é mobilizado, e da pressão promovida pelo trânsito de máquinas e implementos agrícolas, sobretudo quando realizado em solos argilosos e com teores elevados de umidade (Tormena et al., 1998). Contudo, segundo esses autores, com o decorrer do tempo, a densidade do solo sob SPD pode diminuir, devido, em parte, ao aumento do teor de matéria orgânica na camada superficial, melhorando a agregação e a porosidade total do solo.

Parte da MOS é composta pela matéria orgânica leve (MOL), que é uma fração ativa no solo, constituída por resíduos orgânicos parcialmente humificados em vários estádios de decomposição, com tempo de residência no solo que varia de um a cinco anos (Janzen et al., 1992). Além de ser constituída por restos de plantas, a MOL pode apresentar resíduos de animais e microrganismos em diversos estádios de decomposição (Pereira et al., 2010). A MOL pode ser considerada de rápida dinâmica no solo; assim, sua manutenção é fundamental para a sustentabilidade dos sistemas agrícolas, uma vez que representa, em curto e médio prazos, alto potencial para ciclagem de nutrientes (Compton \& Boone, 2002).

O fósforo remanescente (Prem) é uma medida estreitamente correlacionada à capacidade máxima de adsorção e à capacidade-tampão de fosfatos
(Alvarez V. et al., 2000). A MOS desempenha papel ambivalente em relação à disponibilidade de $\mathrm{P}$, já que tanto pode adsorvê-lo quanto bloquear os sítios de adsorção que ocorrem nas superfícies das argilas e óxidos de ferro e alumínio (Ibia \& Udo, 1993). A adoção de sistemas de manejo que propiciem incremento no teor de MOS ou de suas frações pode promover a redução da adsorção de $\mathrm{P}$, pela formação de complexos que bloqueiam os sítios de adsorção na superfície dos óxidos de ferro e de alumínio (Tirloni et al., 2009). De acordo com Pereira et al. (2010), o uso de plantas de cobertura em SPD pode acarretar aumento dos teores de carbono orgânico total (COT) e MOL, com consequente redução na adsorção de fosfatos e aumento dos teores de Prem.

Aregião do Cerrado do Brasil possui características climáticas próprias, como concentração de chuva no verão (1.200 a $1.800 \mathrm{~mm}$ ) e cinco a seis meses de período seco no inverno. Além disso, os solos desse bioma, de maneira geral, possuem baixa fertilidade natural, pois são altamente intemperizados e de caráter ácido. A união desses fatores dificulta a produção de quantidade suficientes de resíduos vegetais para efetiva proteção da superfície do solo contra os processos erosivos e para manutenção dos níveis adequados de carbono orgânico, necessários para a adoção do SPD (Machado \& Silva, 2001; Fidelis et al., 2003; Bolliger et al., 2006). Entretanto, de acordo com Siqueira Neto et al. (2010), o manejo com grande utilização de fertilizantes e pesticidas nas culturas de soja no verão e milho safrinha faz com que o SPD na região do Cerrado gere elevada produção de grãos e de biomassa da planta, aumentando a MOS com o passar dos anos de implantação do SPD.

A partir do exposto, o objetivo deste trabalho foi quantificar a deposição de resíduos vegetais na superfície do solo (RVS), a matéria orgânica leve (MOL) e os estoques de carbono e nitrogênio e fósforo remanescente, em áreas de Cerrado, em Montividiu (GO), sob SPD com diferentes tempos de implantação, em comparação às áreas de Cerrado nativo e pastagem.

\section{MATERIAL E MÉTODOS}

O estudo foi realizado na Fazenda Montividiu Tiuba, que está localizada próxima ao município de Montividiu-GO (S 17 27' 52,2”, W0 51 10' 33,1" e altitude de $890 \mathrm{~m}$ ). A precipitação pluvial anual média da região é de $1.740 \mathrm{~mm}$, com clima tropical quente, Aw segundo Köppen, estações chuvosa e seca bem definidas e relevo predominantemente plano. O solo das áreas de estudo foi classificado como Latossolo Vermelho distroférrico (Embrapa, 2006). 
Foram estudadas quatro áreas de cultivo: pastagem de Brachiaria decumbens (PA), SPD com três anos de implantação com cultivo de soja no verão e pousio na safrinha (SPD3), SPD com 15 anos de implantação com rotação soja no verão milho/sorgo safrinha (SPD15) e SPD com 20 anos de implantação com rotação soja no verão - milho safrinha (SPD20). Também foram coletadas amostras em uma área de Cerrado situada adjacente às áreas de cultivo.

A área de Cerrado está localizada dentro da área de reserva da Fazenda Montividiu Tiúba (S 17 27' 52,2", W0 51 10' 33,1" e altitude de 890 m). A área de pastagem, Brachiaria decumbens (S 17 25' 58,5”, W0 51 09' 39,4" e altitude de $804 \mathrm{~m}$ ), vem sendo cultivada com uma taxa de lotação aproximada de 1,5 unidade animal por hectare. A área de SPD3 (S 17 27' 20,9”, W0 51 10' 16,3" e altitude de 858 m) foi implantada no ano de 1987, com a derrubada do Cerrado e cultivo com pastagem durante 20 anos; após esse período, foi cultivada com arroz por um ano, e há três anos foi adotado o SPD com cultivo de soja. Já a área de SPD15 (S 17 28' 16,8”, W0 51 11'20,4" e altitude de $899 \mathrm{~m}$ ) possui um histórico de 27 anos de cultivo convencional com plantio de soja no verão e milho safrinha, sendo o SPD adotado em 1995. O SPD20 (S 1728 ' 31,7”, W0 51 10' 43,6" e altitude de $898 \mathrm{~m}$ ) possui o mesmo histórico que a área de SPD15, porém a adoção do SPD deu-se em 1990. Atualmente, as áreas de SPD15 e SPD20 vêm sendo cultivadas com soja no verão e milho ou sorgo na safrinha.

A adubação básica das principais culturas dos sistemas agrícolas foi a seguinte: 1) SPD3 e SPD15 a) soja (verão) - $458 \mathrm{~kg} \mathrm{ha}^{-1}$ da fórmula $02-20-20$; b) milho safrinha: $312 \mathrm{~kg} \mathrm{ha}^{-1}$ da formulação $12-15-15$ no plantio e $120 \mathrm{~kg} \mathrm{ha}^{-1}$ de ureia em cobertura aos 25 DAE; e 2) SPD20 - a) soja (verão) - $200 \mathrm{~kg} \mathrm{ha}^{-1}$ da fórmula 02-20-20 + $60 \mathrm{~kg} \mathrm{ha}^{-1}$ de $\mathrm{K}_{2} \mathrm{O}$ e $\mathrm{P}_{2} \mathrm{O}_{5}$ em cobertura; b) milho safrinha: $30 \mathrm{~kg} \mathrm{ha}^{-1} \mathrm{de} \mathrm{N}+$ $60 \mathrm{~kg} \mathrm{ha}^{-1}$ de $\mathrm{P}_{2} \mathrm{O}_{5}+70 \mathrm{~kg} \mathrm{ha}^{-1}$ de $\mathrm{K}_{2} \mathrm{O}$ no sulco e $120 \mathrm{~kg} \mathrm{ha}^{-1}$ de ureia em cobertura aos 25 DAE. O SPD3 recebeu calagem em 2006 e 2010; o SPD15, em 2007; e o SPD20, em 2008. Já a área de PA recebeu uma aplicação de $2 \mathrm{Mg} \mathrm{ha}^{-1}$ de calcário dolomítico no ano de 2009.

Em cada área foi demarcada uma gleba representativa de 2,25 ha $(150 \times 150 \mathrm{~m})$, e em cada uma delas foram abertas cinco trincheiras de aproximadamente $1 \times 1 \mathrm{~m}$ de superfície e $1 \mathrm{~m}$ de profundidade em posição. O delineamento utilizado foi inteiramente casualizado. Em cada uma das trincheiras, nas diferentes áreas, foi realizada a coleta de amostras indeformadas, com auxílio de um anel volumétrico (Embrapa, 1997), nas profundidades de $0-5,5-10$ e $10-20 \mathrm{~cm}$. Também foi determinada a densidade de partículas (Dp) pelo método do balão volumétrico, visando utilizar esses dados em conjunto com a Ds para o cálculo do volume total de poros (VTP), sendo esta calculada pela equação proposta por Vomocil (1965):VTP (\%) $=(1-(\mathrm{Ds} / \mathrm{Dp})) \times 100$.

Nessas mesmas profundidades foram coletadas amostras deformadas, as quais foram secas ao ar, destorroadas e passadas em peneira de $2 \mathrm{~mm}$ de malha, obtendo-se a terra fina seca ao ar (TFSA), na qual foi realizada a caracterização química e análise granulométrica (Embrapa, 1997) (Quadro 1).

A matéria orgânica leve em água (MOL) foi quantificada na profundidade de $0-5 \mathrm{~cm}$, sendo determinada pelo método da flotação em água (Anderson \& Ingram, 1989). Os teores de C foram determinados por combustão seca. A partir dos valores encontrados, foi calculado o conteúdo total de $\mathrm{C}$ na MOL (CONTCMOL). O valor foi obtido pela multiplicação dos teores de $\mathrm{C}$ da MOL pela quantidade de MOL determinada.

A abundância natural do ${ }^{13} \mathrm{C}\left(\delta^{13} \mathrm{C} \%\right.$ ) foi quantificada com o auxílio do espectrômetro de massa Finnigan Delta Plus, no Laboratório de Ecologia Isotópica (CENA-USP), em Piracicaba. Os resultados foram expressos na forma de delta ${ }^{13} \mathrm{C}(\%)$, em relação ao padrão internacional PDB (Belemnitella Americana da formação Pee Dee).

O fósforo remanescente (Prem) foi avaliado na profundidade de $0-5 \mathrm{~cm}$, segundo Alvarez V.\& Fonseca (1990) e Braga \& Defelipo (1974).

Os teores de $\mathrm{C}$ e $\mathrm{N}$ foram quantificados por meio de combustão seca por analisador CHNS (Elementar Analysensysteme $\mathrm{GmbH}$, Hanau, Alemanha). A partir dos dados dos teores de $\mathrm{C}$ e $\mathrm{N}$ e da Ds, foram calculados os estoques de carbono (EstC) e nitrogênio (EsTN) pelo método de massa equivalente (Ellert \& Bettany, 1995; Sisti et al., 2004).

A quantidade de resíduos vegetais depositados na superfície do solo em cada área de estudo foi determinada por meio do lançamento aleatório de um gabarito metálico $\left(0,25 \mathrm{~m}^{2}\right)$ e posterior coleta dos resíduos contidos no gabarito. Em cada área de estudo realizaram-se 20 amostragens. Os resíduos vegetais coletados foram acondicionados em estufa com ventilação forçada, em temperatura de $65{ }^{\circ} \mathrm{C}$, por $72 \mathrm{~h}$; após esse período, realizou-se a pesagem para determinação da massa seca. A partir desses dados, foi determinada a quantidade de massa seca de resíduos vegetais na superfície do solo por hectare.

Para todos os dados, em cada profundidade, foi feita a avaliação da normalidade dos dados (Lilliefors) e da homogeneidade das variâncias dos erros pelo teste de Cochran \& Barttlet. Posteriormente, os resultados foram submetidos à 
Quadro 1. Atributos químicos e análise granulométrica das áreas de Cerrado (CE), de pastagem (PA) e de sistema plantio direto com 3 (SPD3), 15 (SPD15) e 20 (SPD20) anos de implantação

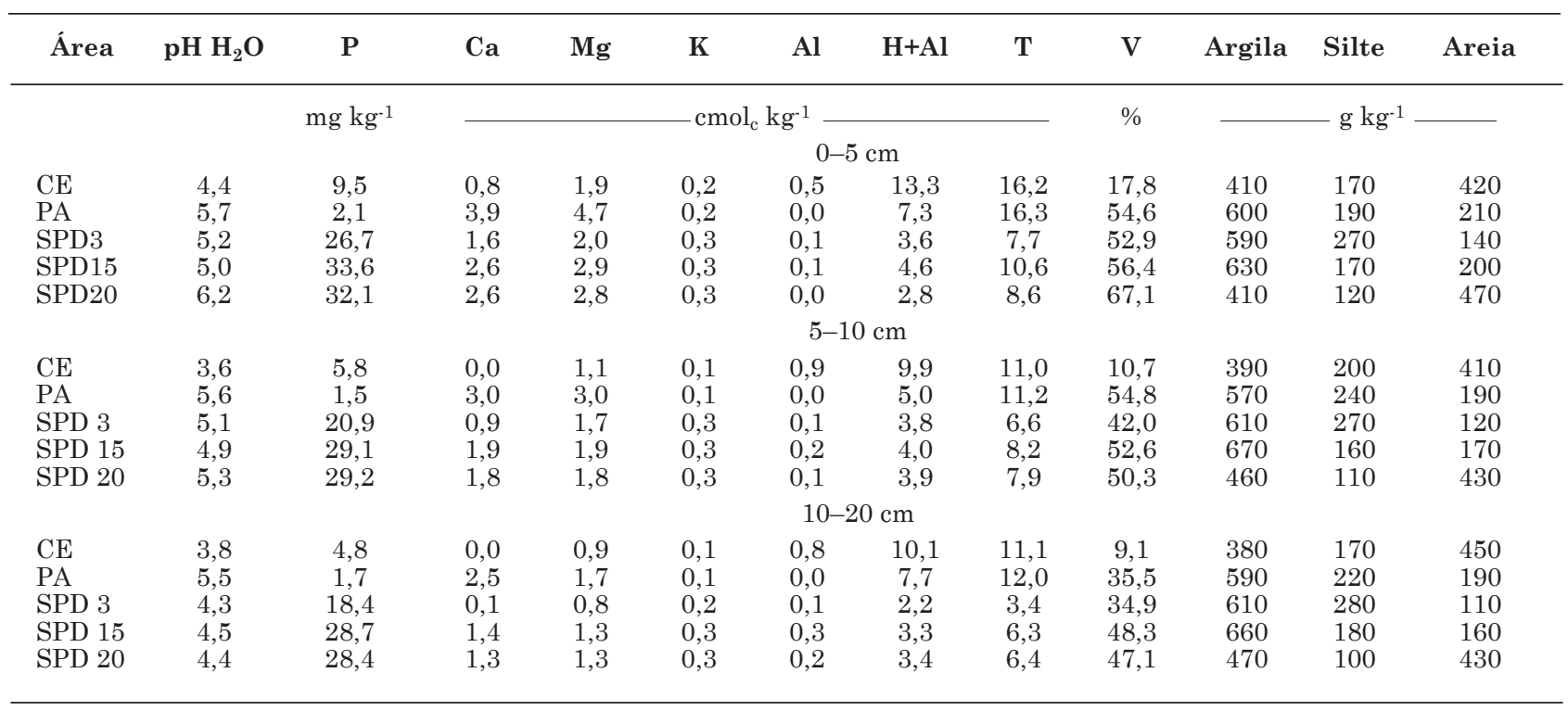

análise de variância com aplicação do teste $\mathrm{F}$, e os valores médios foram comparados entre si pelo teste de Tukey a $5 \%$, com auxílio do programa ASSISTAT (Silva \& Azevedo, 2002).

\section{RESULTADOS E DISCUSSÃO}

\section{Aporte de resíduos vegetais e matéria orgânica leve}

No quadro 2, pode-se verificar que houve diferença estatística na quantidade de resíduos depositados na superfície (RSV), na matéria orgânica leve (MOL), no conteúdo total de C na MOL (CONTCMOL) e ${ }^{13} \mathrm{C}$ da MOL, na camada de $0-5 \mathrm{~cm}$ do solo, nas diferentes áreas.

A maior quantidade de RVS na área de Cerrado é decorrente da deposição contínua de serapilheira, além de essa área não ser submetida a nenhum tipo de ação antrópica. Segundo Moraes et al. (1993), em florestas tropicais, como o Cerrado, cerca de $90 \%$ da produção primária líquida pode ser devolvida ao solo na forma de serapilheira, constituindo um importante reservatório de matéria orgânica e nutrientes. Resultados semelhantes aos deste estudo foram observados por Maman et al. (2007) e Melo $\&$ Resck (2003), os quais, ao avaliarem um Cerrado nativo no sudoeste do Mato Grosso e Planaltina-DF, encontraram produções de 9,4 e $9,5 \mathrm{Mg} \mathrm{ha}^{-1}$ de massa seca de serapilheira, respectivamente.

Em relação às áreas de $\mathrm{SPD}$, observa-se que

Quadro 2. Resíduos vegetais depositados na superfície do solo (RVS), matéria orgânica leve (MOL), carbono da MOL (C-MOL), conteúdo total de $\mathrm{C}$ na MOL (CONTCMOL) e ${ }^{13} \mathrm{C}$ da $\mathrm{MOL}$, na camada de 0-5 cm do solo, nas áreas avaliadas

\begin{tabular}{|c|c|c|c|c|c|}
\hline Sistema avaliado & RVS & MOL & C-MOL & CONTCMOL & ${ }^{13} \mathrm{C}$ \\
\hline & $\mathrm{Mg} \mathrm{ha}^{-1}$ & $\mathrm{~g} \mathrm{~kg}^{-1}$ & $\%$ & $\mathrm{~g} \mathrm{~kg}^{-1}$ & \\
\hline $\mathrm{CE}$ & $10,07 \mathrm{a}^{(1)}$ & $4,21 \mathrm{a}$ & 34,38 & $1,41 \mathrm{a}$ & $-20,85 a$ \\
\hline PA & $7,33 \mathrm{c}$ & $1,23 \mathrm{~b}$ & 32,90 & $0,38 \mathrm{~d}$ & $-14,43 \mathrm{~b}$ \\
\hline SPD3 & $7,32 \mathrm{c}$ & $1,80 \mathrm{~b}$ & 32,79 & $0,56 \mathrm{c}$ & $-21,10 \mathrm{a}$ \\
\hline SPD15 & $7,85 \mathrm{~b}$ & $1,84 \mathrm{~b}$ & 38,69 & $0,70 \mathrm{~b}$ & $-20,55 \mathrm{a}$ \\
\hline SPD20 & $8,65 \mathrm{~b}$ & $4,04 \mathrm{a}$ & 36,28 & $1,47 \mathrm{a}$ & $-20,63 \mathrm{a}$ \\
\hline $\mathrm{CV}(\%)$ & 4,79 & 8,37 & - & 5,39 & 1,35 \\
\hline
\end{tabular}

(1) Médias seguidas de mesma letra na coluna não diferem entre as áreas avaliadas pelo teste de Tukey a $5 \%$. 
onde esse sistema está instalado há mais tempo (15 e 20 anos) existe uma maior produção de resíduos, sendo esta semelhante nas áreas de 15 e 20 anos e superior à área SPD3.

Ao se comparar a área de SPD com a área de Cerrado, verifica-se que mesmo nas áreas onde o SPD está instalado há mais tempo (15 e 20 anos) a produção de resíduos não foi suficiente para que fossem alcançados os valores quantificados na área de Cerrado. Esse resultado pode ser decorrente da maior dificuldade no estabelecimento de plantas de cobertura e consequente baixa produção de biomassa nesse ambiente. De acordo com Fidelis et al. (2003), os principais fatores que interferem na adoção do SPD na região do Cerrado são as características edafoclimáticas, como a concentração de chuva no verão (1.200 a $1.800 \mathrm{~mm}$ ), cinco a seis meses de período seco no inverno e ocorrência de períodos de chuvas estivais, conhecido como veranico.

A baixa produção de resíduos na área de pastagem (PA) em relação ao Cerrado (CE), SPD15 e SPD20 deve-se a um conjunto de fatores, como baixa produtividade, ausência de manejo (principalmente adubação de manutenção) e pastoreio intensivo. O histórico da área de PA demonstra que ela não recebe adubação de manutenção anualmente. De acordo com Zanine et al. (2005), a falta de adubação faz com que haja baixa produtividade da pastagem. Estima-se que $80 \%$ das pastagens cultivadas no Brasil Central, responsáveis por mais de $55 \%$ da produção da carne nacional, encontram-se em algum estádio de degradação, sendo um dos principais motivos a falta de adubação (Macedo et al., 2000).

Os resultados de MOL demonstraram que em função do tempo de adoção do SPD ocorre aumento no aporte desta fração na camada superficial; para as áreas em estudo, após a instalação do SPD por 20 anos, quantificaram-se teores que não diferiram daqueles observados na área de CE. Esse resultado demonstra a grande importância do SPD nesse ambiente, pois a manutenção da MOL é fundamental para a sustentabilidade dos sistemas agrícolas, uma vez que ela representa, em curto e médio prazos, alto potencial para ciclagem de nutrientes (Compton \& Boone, 2002). De acordo com Loss et al. (2010), a MOL é alterada pela forma de manejo da área; em áreas sob SPD, por ser mantida a palhada sobre a superfície do solo, há aumento dos teores da fração leve da MOS. A ausência de diferenças significativas para a MOL entre as áreas de SPD15, SPD3 e PA e os valores menores em relação ao CE e SPD20 podem ser decorrentes do aporte de RVS. Nas áreas SPD15 e SPD3, o tempo de implantação do SPD pode não ter sido suficiente para a modificação desse atributo, ao passo que na área de pastagem (PA), o manejo adotado não favoreceu grandes adições de resíduos e aumentos dos teores de MOL. Avaliando o efeito do plantio de eucalipto na MOL em região de Cerrado no Vale do Jequitinhonha, MG, Pulrolnik et al. (2009) quantificaram maiores teores de MOL nas áreas onde ocorreram as maiores deposições de resíduos.

A MOL foi uma variável mais sensível que os RVSs na estratificação dos sistemas de cultivo avaliados, pois os $9,25 \%$ a mais de RVS do SPD20, mesmo não acarretando diferença em relação ao SPD15, proporcionaram valores de MOL que não diferiram daqueles observados na área e foram superiores aos das demais áreas.

No quadro 2, verifica-se que, quanto maior o tempo de adoção do SPD, maior é o conteúdo de carbono na MOL (CONTCMOL), e que, nas áreas sob SPD, esses valores foram superiores quando comparados aos da área de PA. Pode-se inferir que esses resultados estão relacionados à quantidade e qualidade dos resíduos vegetais que são adicionados ao solo. Os resíduos vegetais com diferentes relações $\mathrm{C} / \mathrm{N}$ aumentam a recalcitrância da MOL, levando a acúmulo de $\mathrm{C}$ em função do tempo de adoção dos sistemas. A área de CE apresentou valores de CONTCMOL semelhantes aos da área de SPD20, e nelas foram verificadas quantidades de CONTCMOL superiores às observadas nas áreas PA, SPD3 e SPD15. Esse padrão também pode ser atribuído à qualidade do material que está originando a MOL na área de CE, pois é comum esse tipo de vegetação apresentar grande diversidade de vegetais, que depositam no solo resíduos orgânicos de diferentes tamanhos e com alta quantidade de lignina (Cianciaruso et al., 2006).

Os resultados da análise de ${ }^{13} \mathrm{C}$ da $\mathrm{MOL}$ (Quadro 2) demonstraram que nas áreas sob SPD e CE essa fração é constituída principalmente por resíduos vegetais de leguminosas, enquanto na área de PA ela é proveniente de gramíneas. Esses dados estão de acordo com o tipo de cobertura observado em cada área, pois sabe-se que na área de CE os resíduos vegetais que dão origem à MOL são provenientes de espécies arbóreas de ciclo fotossintético C3; da mesma forma, as áreas de SPD permanecem a maior parte do ano com cultivo de soja também de ciclo fotossintético C3 (Taiz \& Zeiger, 2004). Já na área de PA, por apresentar histórico de 20 anos de cultivo com gramíneas, verifica-se menor valor de ${ }^{13} \mathrm{C}$ da MOL, visto que gramíneas discriminam menos ${ }^{13} \mathrm{C}$ do que as leguminosas.

\section{Carbono, nitrogênio e $\mathbf{P}$ remanescente do solo}

$\mathrm{Na}$ camada de $0-5 \mathrm{~cm}$ verificaram-se maiores teores de $\mathrm{C}$ e $\mathrm{N}$ em função do tempo de implantação do SPD; para a área de SPD20, quantificaram-se valores de $\mathrm{C}$ e $\mathrm{N}$ superiores aos observados na área de CE (Quadro 3). 
Quadro 3. Carbono total do solo (C), nitrogênio total do solo (N), relação $\mathrm{C} / \mathrm{N},{ }^{13} \mathrm{C}$ do solo e fósforo remanescente (Prem) das áreas avaliadas

\begin{tabular}{|c|c|c|c|c|c|c|}
\hline \multirow{2}{*}{ Camada } & \multicolumn{6}{|c|}{ Sistema avaliado } \\
\hline & $\mathbf{C E}$ & PA & SPD3 & SPD 15 & SPD20 & CV(\%) \\
\hline $\mathrm{cm}$ & \multicolumn{6}{|c|}{$\mathrm{C}\left(\mathrm{g} \mathrm{kg}^{-1}\right)$} \\
\hline $0,0-5,0$ & $30,8 \mathrm{~b}^{(1)}$ & $15,4 \mathrm{c}$ & $15,9 \mathrm{c}$ & $29,6 \mathrm{~b}$ & $33,9 \mathrm{a}$ & 3,1 \\
\hline $5,0-10,0$ & $36,1 \mathrm{a}$ & $14,1 \mathrm{c}$ & $15,4 \mathrm{c}$ & $27,1 \mathrm{~b}$ & $26,7 \mathrm{~b}$ & 2,8 \\
\hline \multirow[t]{2}{*}{$10,0-20,0$} & $31,9 \mathrm{a}$ & $12,04 \mathrm{c}$ & $12,5 \mathrm{c}$ & $25,4 \mathrm{~b}$ & $24,8 \mathrm{~b}$ & 3,7 \\
\hline & \multicolumn{6}{|c|}{$N\left(\mathrm{~g} \mathrm{~kg}^{-1}\right)$} \\
\hline $0,0-5,0$ & $1,9 \mathrm{~b}$ & $1,0 \mathrm{~d}$ & $0,9 \mathrm{~d}$ & $1,7 \mathrm{c}$ & $2,4 \mathrm{a}$ & 3,3 \\
\hline $5,0-10,0$ & $2,2 \mathrm{a}$ & $0,8 \mathrm{e}$ & $0,9 \mathrm{~d}$ & $1,5 \mathrm{c}$ & $1,8 \mathrm{~b}$ & 1,7 \\
\hline \multirow[t]{2}{*}{$10,0-20,0$} & $2,0 \mathrm{a}$ & $0,7 \mathrm{~d}$ & $0,7 \mathrm{~d}$ & $1,4 \mathrm{c}$ & $1,5 \mathrm{~b}$ & 3,1 \\
\hline & \multicolumn{6}{|c|}{${ }^{13} \mathrm{C}\left(\%_{0}\right)$} \\
\hline $0,0-5,0$ & $-25,3 \mathrm{a}$ & $-14,4 \mathrm{~d}$ & $-16,7 \mathrm{c}$ & $-16,6 c$ & $-18,8 \mathrm{~b}$ & 1,4 \\
\hline $5,0-10,0$ & $-25,4 \mathrm{a}$ & $-14,5 \mathrm{e}$ & $-16,0 \mathrm{~d}$ & $-16,3 \mathrm{c}$ & $-18,6 \mathrm{~b}$ & 0,7 \\
\hline \multirow[t]{2}{*}{$10,0-20,0$} & $-25,1 \mathrm{a}$ & $-14,6 \mathrm{~d}$ & $-14,6 \mathrm{~d}$ & $-15,7 \mathrm{c}$ & $-18,0 \mathrm{~b}$ & 1,9 \\
\hline & \multicolumn{6}{|c|}{ Prem (mg kg-1) } \\
\hline $0,0-5,0$ & $15,0 \mathrm{~b}$ & $6,9 \mathrm{e}$ & $11,3 \mathrm{~d}$ & $13,3 \mathrm{c}$ & $16,7 \mathrm{a}$ & 3,7 \\
\hline
\end{tabular}

(1) Médias seguidas de mesma letra minúscula na linha não diferem significativamente entre os diferentes sistemas de uso do solo pelo teste de Tukey a $5 \%$.

Também nessa profundidade observa-se que a área de SPD15 já apresenta teores de C similares aos da área de CE. Esses resultados demonstram que o aporte de materiais em diferentes quantidades e qualidades contribui para o aumento dos teores de C e N. Resultados semelhantes foram encontrados por Matias et al. (2009), os quais verificaram que áreas de SPD em um Latossolo na região de Uruçuí - PI apresentaram aumento nos teores de $\mathrm{C}$ e $\mathrm{N}$ em relação as áreas de Cerrado nativo. Da mesma forma, Corazza et al. (1999), estudando o $\mathrm{C}$ no solo de diferentes sistemas de manejo, em relação às áreas de Cerrado nativo, em um Latossolo Vermelho-Escuro na região de Planaltina-DF constataram que o SPD aumentou o estoque de C em comparação com a área de CE. Segundo esses autores, esse aumento pode ser atribuído à maior taxa de adição de $\mathrm{C}$ ao solo, sendo resultante do aumento da produtividade primária do ecossistema promovido pelo menor revolvimento do solo. O aumento dos teores de $\mathrm{N}$ no solo em áreas de SPD, em relação às áreas de $\mathrm{CE}$, também foi constatado por Buso \& Kliemann (2003), os quais, ao avaliarem a camada de $0-12,5 \mathrm{~cm}$ em um Latossolo Vermelho distrófico na região de Rio Verde - GO, observaram que o SPD proporciona aumento, embora lento, no estoque de $\mathrm{N}$ total do solo em relação ao $\mathrm{CE}$.

Nas demais profundidades analisadas (5-10 e 10-20 cm), a área de CE apresentou os maiores teores de $\mathrm{C}$ e $\mathrm{N}$ em relação aos demais sistemas de cultivo. Já nas áreas de SPD, os teores de C nas áreas de SPD15 e SPD20 não diferiram entre si e foram superiores aos do SPD3, enquanto o teor de
$\mathrm{N}$ aumentou em função do tempo de implantação do SPD. De acordo com Siqueira Neto et al. (2009), o cerradão apresenta maiores quantidades de $\mathrm{C}$ e $\mathrm{N}$ no solo em decorrência do aporte constante de resíduos vegetais e não perturbação do sistema. Em relação às áreas de SPD, observa-se que houve aumento no teor de $\mathrm{C}$ e $\mathrm{N}$ em função do tempo de implantação, porém ele foi menos expressivo que o observado na camada superficial $(0-5 \mathrm{~cm})$. Segundo Bayer \& Mielniczuk (1999), o acúmulo de MOS no SPD ocorre lentamente; assim, pode-se inferir pelos resultados deste trabalho que 20 anos de implantação do SPD ainda não foram capazes de acumular quantidades de $\mathrm{C}$ e $\mathrm{N}$ nas camadas subsuperficiais, quando comparadas à vegetação de CE.

Quanto aos valores de Prem, verificou-se que eles apresentaram padrão semelhante aos dos teores de $\mathrm{C}$ na profundidade de $0-5 \mathrm{~cm}$, ou seja, está ocorrendo aumento no teor de Prem em função do tempo de implantação do SPD; a área de SPD20 foi a que apresentou teores de Prem superiores aos quantificados na área de CE (Quadro 3). Podese inferir que o incremento de matéria orgânica está promovendo a redução da adsorção de P. Uma possível explicação para o padrão observado pode ser o bloqueio dos sítios responsáveis pela adsorção dos íons fosfato pelas substâncias húmicas, reduzindo dessa forma a fixação do $\mathrm{P}$ (Tirloni et al., 2009). Resultados semelhantes foram observados por Pereira et al. (2010) em solos do Cerrado mineiro. Estes autores mencionam que o uso de plantas de cobertura em SPD pode acarretar aumento dos teores de carbono orgânico (CO) total 
e MOL e, consequentemente, diminuir a adsorção de fosfatos e favorecer o aumento nos teores de Prem. De maneira geral, e independentemente da profundidade analisada, as áreas de PA e de SPD3 apresentaram os menores valores de C, N e Prem em relação às demais. Os resultados observados nestas áreas (SPD3 e PA) devem-se ao pequeno tempo de implantação do SPD e, na área de pastagem, ao fato de esta ser de pastoreio intensivo e ausência de manejo (calagem e adubação). $\mathrm{Na}$ área de $\mathrm{PA}$, observa-se baixo aporte de resíduos vegetais, o que contribui para os menores valores de $\mathrm{C}, \mathrm{N}$ e, consequentemente, Prem.

Verifica-se que existe relação direta entre os dados de ${ }^{13} \mathrm{C}$ do solo (Quadro 3) e de ${ }^{13} \mathrm{C}$ da MOL (Quadro 2). A área de CE onde foi quantificado o maior aporte de resíduos vegetais oriundos de plantas C3 na superfície do solo apresentou valores de ${ }^{13} \mathrm{C}$ na MOL de $-20 \%$ e, no solo, de $-25 \%$ o, os quais estão próximos dos estabelecidos para plantas C3 (-28 \%o). Da mesma maneira, a área de PA, por ter recebido aporte de resíduos vegetais oriundos, basicamente, de gramíneas, apresentou valores de ${ }^{13} \mathrm{C}$ na MOL de $-14 \%$ o e, no solo, de - $14 \%$ - próximos aos observados para plantas C4 (-12\%o).

As áreas de SPD recebem, durante o ano, resíduos tanto de leguminosas como de gramíneas, porém, como já discutido, o sinal de ${ }^{13} \mathrm{C}$ da $\mathrm{MOL}$ dessas áreas indicou maior contribuição de plantas C3 (leguminosas). Assim, observa-se que, em razão do tempo de implantação do SPD, o sinal negativo de ${ }^{13} \mathrm{C}$ do solo indica que os valores dessa variável estão se reduzindo em todas as profundidades analisadas, ou seja, estão se modificando em direção aos valores médios estabelecidos para plantas C3 (-28\%o).

\section{Densidade do solo, densidade de partícula e volume total de poros}

Não foram verificadas diferenças para a Dp entre as áreas de estudo. Os valores de Dp estiveram entre 2,59 e 2,64 $\mathrm{Mg} \mathrm{m}^{-3}$ (Quadro 4), o que sugere que a composição mineralógica das frações areia e argila é constituída principalmente por quartzo e caulinita, respectivamente, já que esses minerais possuem valor médio de massa específica de $2,65 \mathrm{Mg} \mathrm{m}^{-3}$.

Os menores valores de Ds, bem como os maiores valores de VTP, na área de CE em comparação às áreas de cultivo (PA, SPD3 e SPD15) podem ser atribuídos ao maior acúmulo de resíduos vegetais na superfície do solo e ao maior acúmulo de $\mathrm{C}$ nas camadas superficiais, bem como à menor alteração antrópica (Quadro 4). Resultados semelhantes foram observados por Bayer et al. (2006) e Carneiro et al. (2009), que, ao avaliarem sistemas de manejo em Latossolos no Estado de Goiás, constataram
Quadro 4. Densidade do solo (Ds), densidade de partículas (Dp) e volume total de poros (VTP) das áreas avaliadas

\begin{tabular}{|c|c|c|c|}
\hline \multirow{2}{*}{ Áreas } & \multicolumn{3}{|c|}{ Camada (cm) } \\
\hline & $0-5$ & $5-10$ & $10-20$ \\
\hline & \multicolumn{3}{|c|}{$\operatorname{Ds}\left(\mathrm{Mg} \mathrm{m}^{-3}\right)$} \\
\hline $\mathrm{CE}$ & $0,88 b^{(1)}$ & $1,06 \mathrm{~b}$ & $1,13 \mathrm{~b}$ \\
\hline PA & $1,20 \mathrm{a}$ & $1,20 \mathrm{a}$ & $1,22 \mathrm{a}$ \\
\hline SPD3 & $1,18 \mathrm{a}$ & $1,18 \mathrm{a}$ & $1,21 \mathrm{a}$ \\
\hline SPD15 & $1,18 \mathrm{a}$ & $1,23 \mathrm{a}$ & $1,26 \mathrm{a}$ \\
\hline SPD20 & $1,05 \mathrm{~b}$ & $1,07 \mathrm{~b}$ & $1,13 \mathrm{~b}$ \\
\hline \multirow[t]{2}{*}{ CV (\%) } & 2,38 & 4,53 & 5,18 \\
\hline & \multicolumn{3}{|c|}{$\mathrm{Dp}\left(\mathrm{Mg} \mathrm{m}^{-3}\right)$} \\
\hline $\mathrm{CE}$ & $2,62 \mathrm{a}$ & $2,62 \mathrm{a}$ & $2,63 \mathrm{a}$ \\
\hline PA & $2,59 \mathrm{a}$ & $2,61 \mathrm{a}$ & $2,61 \mathrm{a}$ \\
\hline SPD3 & $2,59 \mathrm{a}$ & $2,62 \mathrm{a}$ & $2,62 \mathrm{a}$ \\
\hline SPD15 & $2,61 \mathrm{a}$ & $2,62 \mathrm{a}$ & $2,62 \mathrm{a}$ \\
\hline SPD20 & $2,61 \mathrm{a}$ & $2,62 \mathrm{a}$ & $2,64 \mathrm{a}$ \\
\hline \multirow[t]{2}{*}{ CV (\%) } & 1,01 & 0,96 & 0,89 \\
\hline & \multicolumn{3}{|c|}{ VTP (\%) } \\
\hline $\mathrm{CE}$ & $66,40 \mathrm{a}$ & $60,13 \mathrm{a}$ & $56,70 \mathrm{a}$ \\
\hline PA & $53,68 \mathrm{c}$ & $53,72 \mathrm{~b}$ & $53,04 \mathrm{~b}$ \\
\hline SPD3 & $54,75 \mathrm{c}$ & $54,49 \mathrm{~b}$ & $53,78 \mathrm{~b}$ \\
\hline SPD15 & $54,60 \mathrm{c}$ & $52,57 \mathrm{~b}$ & $51,40 \mathrm{c}$ \\
\hline SPD20 & $58,88 \mathrm{~b}$ & 59,27 a & $57,19 \mathrm{a}$ \\
\hline CV (\%) & 1,78 & 1,27 & 0,92 \\
\hline
\end{tabular}

(1) Médias seguidas de mesma letra minúscula na coluna não diferem significativamente entre os diferentes sistemas de uso do solo pelo teste de Tukey a $5 \%$.

que a área de Cerrado foi a que apresentou os menores valores de Ds. Os menores valores de Ds, bem como os maiores valores de VTP, na área de SPD20 em relação às demais áreas de SPD podem ser explicados pelo fato de nos primeiros anos de SPD ocorrer aumento na Ds pela reorganização das partículas de solo; no entanto, com a consolidação do sistema, há aumento nos teores de carbono orgânico, o que leva a redução na Ds e consequente aumento no VTP (Tormena et al., 1998). Já em relação à área de PA, os maiores valores de Ds em relação às áreas de CE e SPD20 podem ser decorrentes do menor conteúdo de $\mathrm{C}$, bem como do efeito do pastoreio excessivo a que essa área é submetida.

Nota-se que o CE, PA, SPD3, SPD15 e SPD20 apresentam, até os $20 \mathrm{~cm}$ do solo, Ds abaixo do limite crítico (1,30 a 1,40 - solo argiloso e 1,26 a $1,30 \mathrm{Mg} \mathrm{m}^{-3}$ - solo muito argiloso); assim, pode-se inferir que a Ds não está prejudicando o desenvolvimento das culturas implantadas nessas áreas (Reichert et al., 2003). 


\section{Estoque de carbono e nitrogênio do solo}

No quadro 5, pode-se verificar que as áreas de PA e SPD3 apresentaram os menores valores de estoque de carbono (EstC) e estoque de nitrogênio (EstN) em relação aos demais sistemas de cultivo, em todas as profundidades. Observa-se também que nas áreas sob SPD15 e SPD20, nas camadas superficiais, os valores de EstC não diferiram entre si e daqueles da área de CE, porém nas camadas de 10-20 e 0-20 cm percebe-se que as áreas mais antigas de SPD mostraram valores de EstC inferiores ao CE.

Observa-se aumento do EstN em função do tempo de adoção do SPD em todas as profundidades analisadas, sendo verificado que nas camadas superficiais da área com SPD20 os valores de EstN são superiores $(0-5 \mathrm{~cm})$ e similares $(5-10 \mathrm{~cm})$ aos da área de CE (Quadro 5).

Uma possível explicação para os baixos valores de EstC e EstN no SPD3, em relação aos demais sistemas de cultivo, pode ser o pequeno tempo de adoção do sistema, visto que, segundo Carvalho et al. (2009), o aumento de MOS em áreas de SPD em comparação a outros sistemas de manejo pode estar relacionado principalmente ao seu tempo de instalação, pois o acúmulo de $\mathrm{C}$ nesse sistema ocorre muito lentamente, levando de 10 a 15 anos para se tornar expressivo. Já os baixos teores de EstC e EstN da PA podem estar relacionados à sua baixa produtividade de resíduos vegetais, à ausência de manejo e pastoreio intensivo, ao pequeno aporte de resíduos vegetais e, também, à redução na distribuição do sistema radicular. Silva et al. (2004) também verificaram que pastagens de baixa produtividade, em diversas regiões do Cerrado, favoreceram a redução do teor de $\mathrm{C}$ no solo.

As áreas com 15 e 20 anos de SPD recebem aporte constante de resíduos vegetais de diferentes qualidades, o que pode ter sido um fator preponderante para os valores de EstC serem semelhantes aos observados na área de CE. Resultados idênticos foram relatados por Siqueira Neto et al. (2010), os quais, ao avaliarem no Cerrado áreas com diferentes anos de implantação do SPD em um Latossolo, constataram que aquelas com 12 anos de cultivo apresentaram valores de EstC semelhantes aos do Cerrado. Esses autores atribuíram o resultado à grande utilização de fertilizantes e pesticidas nas culturas de soja no verão e milho safrinha, que favorecem maiores produções de grãos e biomassa, aumentando a MOS em função do tempo de implantação do SPD.

$\mathrm{Na}$ profundidade de $10-20 \mathrm{~cm}$ e na soma das camadas de 0-20 cm, a área de $\mathrm{CE}$ apresentou os maiores valores de EstC em relação aos sistemas de cultivo, enquanto as áreas de SPD15 e SPD20 foram estatisticamente semelhantes e superiores às áreas de SPD3 e PA. De acordo com Siqueira Neto et al. (2009), o cerradão apresenta maiores quantidades de $\mathrm{C}$ e $\mathrm{N}$ no solo em decorrência do aporte constante de resíduos vegetais e não perturbação do sistema. No tocante às áreas de SPD, observa-se que há aumento no teor de $\mathrm{C}$ e $\mathrm{N}$ em função do tempo de implantação, porém ele é menos expressivo que o observado nas camadas superficiais do solo (0-5 e 5-10 cm). Segundo Bayer \& Mielniczuk (1999), o acúmulo de MOS no SPD ocorre lentamente; assim, pode-se inferir que 15 e 20 anos de implantação do

Quadro 5. Estoque de carbono (EstC) e de nitrogênio (EstN) das áreas avaliadas

\begin{tabular}{|c|c|c|c|c|c|c|}
\hline \multirow{2}{*}{ Camada } & \multicolumn{6}{|c|}{ Sistema avaliado } \\
\hline & CE & PA & SPD3 & SPD 15 & SPD20 & CV(\%) \\
\hline $\mathrm{cm}$ & \multicolumn{6}{|c|}{ EstC (Mg ha-1) } \\
\hline $0,0-5,0$ & $13,54 \mathrm{a}^{(1)}$ & $6,78 \mathrm{~b}$ & $7,04 \mathrm{~b}$ & $13,07 \mathrm{a}$ & $14,96 \mathrm{a}$ & 7,12 \\
\hline $5,0-10,0$ & $17,86 \mathrm{a}$ & $7,22 \mathrm{~b}$ & $7,71 \mathrm{~b}$ & $13,78 \mathrm{a}$ & $13,90 \mathrm{a}$ & 15,60 \\
\hline $10,0-20,0$ & $36,34 \mathrm{a}$ & $14,28 \mathrm{c}$ & $14,98 \mathrm{c}$ & $29,45 \mathrm{~b}$ & $28,53 \mathrm{~b}$ & 3,81 \\
\hline \multirow[t]{2}{*}{$0,0-20,0$} & $67,74 \mathrm{a}$ & $28,27 \mathrm{c}$ & $29,72 \mathrm{c}$ & $56,29 \mathrm{~b}$ & $57,39 \mathrm{~b}$ & 2,60 \\
\hline & \multicolumn{6}{|c|}{$\operatorname{EstN}\left(\mathrm{Mg} \mathrm{ha}^{-1}\right)$} \\
\hline $0,0-5,0$ & $0,87 \mathrm{~b}$ & $0,44 \mathrm{c}$ & $0,44 \mathrm{c}$ & $0,77 \mathrm{~b}$ & $1,07 \mathrm{a}$ & 7,89 \\
\hline $5,0-10,0$ & $1,09 \mathrm{a}$ & $0,45 \mathrm{c}$ & $0,47 \mathrm{c}$ & $0,78 \mathrm{~b}$ & $0,94 \mathrm{ab}$ & 14,74 \\
\hline $10,0-20,0$ & $2,29 \mathrm{a}$ & $0,85 \mathrm{c}$ & $0,91 \mathrm{c}$ & $1,64 \mathrm{~b}$ & $1,77 \mathrm{~b}$ & 3,63 \\
\hline $0,0-20,0$ & $4,25 \mathrm{a}$ & $1,74 \mathrm{~d}$ & $1,82 \mathrm{~d}$ & $3,18 \mathrm{c}$ & $3,78 \mathrm{~b}$ & 2,07 \\
\hline
\end{tabular}

(1) Médias seguidas de mesma letra minúscula na linha não diferem significativamente entre os diferentes sistemas de uso do solo pelo teste de Tukey a $5 \%$. 
SPD ainda não foram capazes de acumular C e N nas camadas subsuperficiais, quando comparadas à área de $\mathrm{CE}$.

$\mathrm{O}$ aumento do EstN em função do tempo de implantação do SPD pode estar ocorrendo devido aos elevados teores de argila e adubação nitrogenada das áreas com SPD, onde os valores observados de EstN são similares aos verificados na área de Cerrado (Matias et al., 2009). O EstN na profundidade de 10-20 cm apresentou padrão similar ao observado para o EstC, porém na soma das camadas $(0-20 \mathrm{~cm})$ percebe-se que o EstN foi uma variável mais sensível que o EstC para demonstrar as modificações nos atributos do solo em função do tempo de implantação do SPD, pois as áreas de 15 e 20 anos apresentaram diferenças no EstN mesmo com valores de EstC semelhantes.

\section{CONCLUSÕES}

1. O manejo do solo sob SPD após 20 anos apresentou maiores valores de Prem, C, N e EstN na camada superficial do solo em relação à área de CE. Além disso, o SPD após 20 anos recuperou os valores de Ds, MOL, VTP e EstC, alcançando padrões similares aos quantificados na área de Cerrado.

2. O SPD aumentou os valores de EstC e EsTN em função do tempo de sua implantação em todas as camadas analisadas; as áreas sob SPD15 e SPD20 apresentaram, na camada superficial do solo, valores semelhantes e, ou, superiores aos da área de $\mathrm{CE}$.

3. As análises de ${ }^{13} \mathrm{C}$ demonstraram que as leguminosas estão contribuindo de forma significativa para a composição da MOS nas áreas sob SPD.

4. A área de pastagem apresentou os piores índices das variáveis analisadas neste estudo, demonstrando estar em estádio avançado de degradação.

\section{LITERATURA CITADA}

ALVAREZ V., V.H. \& FONSECA, D.M. Definição de doses de fósforo para determinação da capacidade máxima de adsorção de fosfatos e para ensaios em casa de vegetação. R. Bras. Ci. Solo, 14:49-55, 1990.

ALVAREZ V., V.H.; NOVAIS, R.F.; DIAS, L.E. \& OLIVEIRA, J.A. Determinação e uso do fósforo remanescente. B. Inf. SBCS, 25:27-32, 2000.
ANDERSON, J.N. \& INGRAM, J.S.I. Tropical soil biology and fertility: A handbook of methods. Wallingford, CAB International, 1989. 171p.

BAYER, C. \& MEILNICZUK, J. Dinâmica e função da matéria orgânica. In: SANTOS, G.A. \& CAMARGO, F.A.O., eds. Fundamentos da matéria orgânica do solo, ecossistemas tropicais e subtropicais. Porto Alegre, Genesis, 1999. p.1-26.

BAYER, C.; MARTIN-NETO, L.; MIELNICZUK, J.; PAVINATO, A. \& DIECKOW, J. Carbon sequestration in two Brazilian Cerrado soils under no-till. Soil Tillage Res., 86:237-245, 2006.

BOLLIGER, A.; MAGID, J.; AMADO, T.J.C.; NETO, F.S.; SANTOS RIBEIRO, M.F.; CALEGARI, A.; RALISCH, R. \& NEERGAARD, A. Taking stock of the Brazilian "Zero Till Revolution”. Adv. Agron., 91:47-110, 2006.

BRAGA, J.M. \& DEFELIPO, B.V. Determinação espectrofotométrica de fósforo em extratos de solo e material vegetal. R. Ceres, 21:73-85, 1974.

BUSO, W.H.D. \& KLIEMANN, H.J. Relações de carbono orgânico e de nitrogênio total e potencialmente mineralizável com o nitrogênio absorvido pelo milheto. Pesq. Agropec. Trop., 33:97-105, 2003.

BUSTAMANTE, M.M.C.; CORBEELS, M.; SCOPEL, E. \& ROSCOE, R. Soil carbon and sequestration potential in the Cerrado Region of Brazil. In: LAL, R.; CERRI, C.C.; BERNOUX, M.; ETCHEVERS, J. \& CERRI, C.E.P., eds. Carbon sequestration in soils of Latin America. New York, Haworth, 2006. p.285-304.

CARVALHO, J.L.N.; AVANZI, J.C.; SILVA, M.L.N.; MELLO, C.R. \& CERRI, C.E.P. Potencial de sequestro de carbono em diferentes biomas do Brasil. R. Bras. Ci. Solo, 34:277$289,2010$.

CARVALHO, J.L.N.; CERRI, C.E.P.; FEIGL, B.J.; PÍCCOLO, M.C.; GODINHO, V.P. \& CERRI, C.C. Carbon sequestration in agricultural soils in the Cerrado region of the Brazilian Amazon. Soil Tillage Res., 103:342-349, 2009.

CARNEIRO, M.A.C.; SOUZA, E.D.; REIS, E.F.; PEREIRA, H.S. \& AZEVEDO, W.R. Atributos físicos, químicos e biológicos de solo de Cerrado sob diferentes sistemas de uso e manejo. R. Bras. Ci. Solo, 33:147-157, 2009.

CIANCIARUSO, M.V.; PIRES, J.S.R.; DELITTI, W.B.C. \& SILVA, E.F.L.P. Produção de serapilheira e decomposição do material foliar em um cerradão na Estação Ecológica de Jataí, município de Luiz Antônio, SP, Brasil. Acta Bot. Bras, 20:49-59, 2006.

COMPTON, J.E. \& BOONE, R.D. Soil nitrogen transformations and the role of light fraction organic matter in forest soils. Soil Biol. Biochem., 34:933-943, 2002.

CORAZZA, E.J.; SILVA, J.E.D.; RESCK, D.V.S. \& GOMES, A.C. Comportamento de diferentes sistemas de manejo como fonte ou depósito de carbono em relação à vegetação de Cerrado. R. Bras. Ci. Solo, 23:425-432, 1999.

ELLERT, B.H. \& BETTANY, J.R. Calculation of organic matter and nutrients stored in soils under contrasting management regimes. Can. J. Soil Sci., 75:529-538, 1995. 
EMPRESA BRASILEIRA DE PESQUISA AGROPECUÁRIA EMBRAPA. Centro Nacional de Pesquisa do Solo. Sistema brasileiro de classificação de solos. 2.ed. Rio de Janeiro, 2006. 306p

EMPRESA BRASILEIRA DE PESQUISA AGROPECUÁRIA EMBRAPA. Manual de métodos de análise de solos. Rio de Janeiro, 1997. 212p.

FIDELIS, R.R.; ROCHA, R.N.C.; LEITE, U.T. \& TANCREDI, F.D. Alguns aspectos para do plantio direto para a cultura da soja. Biosci. J., 19:23-31, 2003.

FRANCHINI, J.C.; CRISPINO, C.C.; SOUZA, R.A.; TORRES, E. \& HUNGRIA, M. Microbiological parameters as indicators of soil quality under various soil management and crop rotation systems in southern Brazil. Soil Tillage Res., 92:18-29, 2007.

FOLEY, J.A.; DEFRIES, R.; ASNER, G.P.; BARFORD, C.; BONAN, G.; CARPENTER, S.R.; CHAPIN, F.S.; COE, M.T.; DAILY, G.C.; GIBBS, H.K.; HELKOWSKI, J.H.; HOLLOWAY, T.; HOWARD, E.A.; KUCHARIK, C.J.; MONFREDA, C.; PATZ, J.A.; PRENTICE, I.C.; RAMANKUTTY, N. \& SNYDER, P.K. Global consequences of land use. Science, 309:570-574, 2005.

IBIA, T.O. \& UDO, E.J. Phosphorus forms and fixation capacity of representative soils in Akwa Ibom State of Nigeria. Geoderma, 58:95-106, 1993.

JANZEN, H.H.; CAMPBELL, C.A.; BRANDT, S.A.; LAFOND, G.P. \& TOWNLEY-SMITH, L. Light-fraction organic matter in soils from long-term crop rotations. Soil Sci. Soc. Am. J., 56:1799-1806, 1992.

LOSS, A.; MORAES, A.G.L.; PEREIRA, M.G.; SILVA, E.M.R. \& ANJOS, L.H.C. Carbono, matéria orgânica leve e frações oxidáveis do carbono orgânico sob diferentes sistemas de produção orgânica. Comunicata Sci., 1:57-64, 2010.

MACEDO, M.C.M.; KICHEL, A.N. \& ZIMMER, A.H. Degradação e alternativas de recuperação e renovação de pastagens. Campo Grande, Embrapa/CNPGC, 2000. 4p.

MACHADO, P.O.L.A. \& SILVA, C.A. Soil management under no-tillage systems in the tropics with special reference to Brazil. Nutr. Cycling Agroecosyst., 61:119-130, 2001.

MAMAN, A.P.; SILVA, C.J.; SGUAREZI, E.M. \& BLEICH, M.E. Produção e acúmulo de serapilheira e decomposição foliar em mata de galeria e cerradão no sudoeste de mato grosso. R. Ci. Agro-Amb., 5:71-84, 2007.

MATIAS, M.C.B.S.; SALVIANO, A.A.C.; LEITE, L.F.C. \& ARAÚJO, A.S.F. Biomassa microbiana e estoques de C e $\mathrm{N}$ do solo em diferentes sistemas de manejo, no Cerrado do Estado do Piauí. Acta Sci., 31:517-521, 2009.

MELO, J.T. \& RESCK, D.V.S. Retorno ao solo de nutrientes de serrapilheira de Eucalyptus camaldulensis no Cerrado do Distrito Federal. Planaltina: Embrapa Cerrados, 2003. 17p. (Boletim de Pesquisa e Desenvolvimento)

MELLO, F.F.C.; CERRI, C.E.P.; BERNOUX, M.; VOLKOFF, B. \& CERRI, C.C. Potential of soil carbon sequestration for the Brazilian Atlantic Region. In: LAL, R.; CERRI, C.C.; BERNOUX, M.; ETCHEVERS, J. \& CERRI, C.E.P., eds. Carbon sequestration in soils of Latin America. New York, Haworth, 2006. p.349-368.
MORAES, R.M.; RABELO, C.F.; DELITI, W.B.C. \& VUONO, Y.S. Serapilheira acumulada em um trecho de mata atlântica de encosta, no Parque Estadual da Ilha do Cardoso. In: SIMPÓSIO DE ECOLOGIA DA COSTA BRASILEIRA, 3., 1993, São Paulo. Anais... São Paulo, ACIESP, 1993. p.94-99.

OORTS, K.; VANLAUWE, B. \& MERCKX, R. Cation exchange capacities of soil organic matter fractions in a Ferri Lixisol with different organic matter inputs. Agric. Ecosyst. Environ., 100:161-171, 2003.

PEREIRA, M.G.; LOSS, A.; BEUTLER, S.J. \& TORRES, J.L.R. Carbono, matéria orgânica leve e fósforo remanescente em diferentes sistemas de manejo do solo. Pesq. Agropec. Bras., 45:508-514, 2010.

PULROLNIK, K.; BARROS, N.F.; SILVA, I.R.; NOVAIS, R.F. \& BRANDANI, C.B. Estoques de carbono e nitrogênio em frações lábeis e estáveis da matéria orgânica de solos sob eucalipto, pastagem e Cerrado no vale do Jequitinhonha MG. R. Bras. Ci. Solo, 33:1125-1136, 2009.

REICHERT, J.M.; REINERT, D.J. \& BRAIDA, J.A. Qualidade dos solos e sustentabilidade de sistemas agrícolas. Ci. Amb., 27:29-48, 2003.

SANO, E.E.; ROSA, R.; BRITO, J.L. \& FERREIRA, L.G. Mapeamento semidetalhado do uso da terra do bioma Cerrado. Pesq. Agropec. Bras., 43:153-156, 2008.

SILVA, J.E. \& RESCK, D.V.S. Matéria orgânica do solo. In: VARGAS, M.A.T. \& HUNGRIA, M., eds. Biologia dos solos dos cerrados. Planaltina, Embrapa-CPAC, 1997. p.467-524.

SILVA, F.A.S. \& AZEVEDO, C.A.V. Versão do programa computacional Assistat para o sistema operacional Windows. R. Bras. Produc. Agroindus., 04:71-78, 2002.

SILVA, J.E.; RESCK, D.V.S.; CORAZZA, E.J. \& VIVALDI, L. Carbon storage in clayey oxisol cultivated pastures in the "Cerrado" region, Brazil. Agric. Ecosyst. Environ., 103:357-363, 2004.

SIQUEIRA NETO, M.; PICCOLO, M.C.; SCOPEL, E.; COSTA JUNIOR, C.; CERRI, C.C. \& BERNOUX, M. Carbono total e atributos químicos com diferentes usos do solo no Cerrado. Acta Sci. Agron., 31:709-717, 2009.

SIQUEIRA NETO, M.; SCOPEL, E.; CORBEELS, M.; CARDOSO, A.N.; DOUZET, J.M.; FELLER, C.; PICCOLO, M.C.; CERRI, C.C. \& BERNOUX, M. Estoques de carbono do solo sob plantio direto em sistemas de cultivo no Cerrado do Brasil: Uma avaliação sincrônica na exploração. Soil Tillage Res., 110:187-195, 2010.

SISTI, C.P.J.; SANTOS, H.P.; KOHHANN, R.; ALVES, B.J.R.; URQUIAGA, S. \& BODDEY, R.M. Change in carbon and nitrogen stocks in soil under 13 years of conventional or zero tillage in southern Brazil. Soil Tillage Res., 76:39-58, 2004.

SIX, J.; FELLER, C.; DENEF, K.; OGLE, S.M.; MORAES, J.C. \& ALBRECHT, A. Soil organic matter, biota and aggregation in temperate and tropical soils - Effects of no-tillage. Agronomie, 22:755-775, 2002.

SMITH, P.; JANZEN, H.; MARTINO, D.; ZUCONG, Z.; KUMAR, P.; MCCARL, B.A.; OGLE, S.; O'MARA, F.; RICE, C.; SCHOLES, B.; SIROTENKO, O.; HOWDEN, M.; MCALLISTER, T.; GENXING, P.; ROMANEKOV, V.; SCHNEIDER, U.A.; TOWPRAYOON, S.; WATTENBACH, M. \& SMITH, J. Greenhouse gas mitigation in agriculture. Philos. Trans. Royal Soc., 363:789-813, 2008. 
STONE, L.F. \& SILVEIRA, P.M. Efeitos do sistema de preparo e da rotação de culturas na porosidade e densidade do solo. R. Bras. Ci. Solo, 25:395-401, 2001.

TAIZ, L. \& ZEIGER, E. Fisiologia vegetal. 3.ed. Porto Alegre, Artmed, 2004. 719p.

TIRLONI, C.; VITORINO, A.C.T.; NOVELINO, J.O.; TIRLONI, D. \& COIMBRA, D.S. Disponibilidade de fósforo em função das adições de calagem e de um bioativador do solo. Ci. Agrotecnol., 33:977-984, 2009.

TORMENA, C.A.; ROLOFF, G. \& SÁ, J.C.M. Propriedades físicas do solo sob plantio direto influenciadas por calagem, preparo inicial e tráfego. R. Bras. Ci. Solo, 22:301-309, 1998.
VOMOCIL, J.A. Porosity. In: BLACK, C.A., ed. Methods of soil analysis: Physical and mineralogical properties, including statistics of measurement and sampling. Madison, American Society of Agronomy, 1965. Part 1. p.499-510.

ZANINE, A.M.; SANTOS, E.M. \& FERREIRA, D.J. Possíveis causas da degradação de pastagens. R. Electr. Veter., 06:01-23, 2005. 